\title{
Core biopsy: uma técnica confiável para o diagnóstico histopatológico do câncer de mama?
}

Core biopsy: is this a reliable technique for the histopathological diagnosis of breast cancer?

Alfredo Ribeiro-Silva

O câncer de mama é o tumor maligno mais prevalente entre as mulheres. Segundo a American Cancer Society, cerca de 230 mil novos casos de carcinoma mamário invasor foram previstos para o ano de 2011 nos Estados Unidos, assim como cerca de 39 mil óbitos decorrentes da doença ${ }^{(2)}$. De acordo com dados do Instituto Nacional do Câncer (INCA), a estimativa é que em 2012 sejam diagnosticados no Brasil 52.680 novos casos de câncer de mama ${ }^{(5)}$. O diagnóstico desse câncer depende da interação entre cirurgião, radiologista e patologista; este tendo o papel de destaque nessa equipe multidisciplinar, uma vez que compete a ele o diagnóstico definitivo.

Atualmente, os métodos mais utilizados pelos patologistas para o diagnóstico inicial do câncer de mama são a biópsia por agulha grossa e a punção biópsia aspirativa. Essas duas técnicas possuem vantagens e limitações, mas a biópsia por agulha grossa, mais comumente conhecida por core biopsy, tem sido cada vez mais adotada como procedimento padrão para o diagnóstico inicial do câncer de mama, pois, além de ser um procedimento relativamente pouco agressivo, possibilita análise histopatológica do tumor, ao contrário da punção por agulha fina, que possibilita apenas a análise citopatológica do material obtido. Além disso, dados de literatura indicam que a core biopsy possui sensibilidade e especificidade superiores às da punção biópsia aspirativa, tanto para o diagnóstico de lesões benignas quanto para o diagnóstico de lesões malignas ${ }^{(1)}$.

Uma grande preocupação em relação a core biopsy, entretanto, é a questão da amostragem da lesão. Será que um pequeno cilindro de tecido mamário é representativo da lesão como um todo? Em outras palavras, será que o diagnóstico feito pela core biopsy é confiável? Para tentar contribuir na resposta dessa questão, Galhardo e colaboradores, em artigo publicado nessa edição do Jornal Brasileiro de Patologia e Medicina Laboratorial(3), avaliaram o grau de concordância entre o diagnóstico feito pela core biopsy e a análise histopatológica posterior feita na peça cirúrgica, considerada padrão-ouro. Os autores verificaram que a taxa de concordância em relação ao tipo histológico foi de 76\%. Segundo a literatura, a taxa de concordância é bastante variável entre os diversos estudos, provavelmente devido às diferentes metodologias empregadas, mas, no geral, a taxa de concordância em relação ao tipo histológico é alta, podendo chegar a 97\%, sobretudo para o diagnóstico de lesões malignas( ${ }^{(4)}$.

Todavia, poucos estudos avaliaram a taxa de concordância da core biopsy em relação ao grau histológico e a invasão linfovascular, e essa, com certeza, é a principal contribuição do trabalho de Galhardo 
et $a l^{(3)}$. Esses autores verificaram que a taxa de concordância em relação ao grau histológico foi de apenas $50 \%$, com a core biopsy subestimando o grau. O grau de concordância em relação à invasão linfovascular foi de $62 \%$, com a core biopsy apresentando alta especificidade, mas baixa sensibilidade. Para qualquer procedimento diagnóstico, um valor preditivo positivo alto, como verificado em relação ao diagnóstico de invasão linfovascular na core biopsy, é importante para minimizar a possibilidade de tratamentos agressivos desnecessários.

Os autores concluem que, embora a core biopsy seja um bom método para se diagnosticar o câncer de mama, ela tende a subestimar a agressividade do tumor, principalmente em relação ao grau tumoral e a invasão linfovascular.

\section{Referências}

1. BARRA, A. D. E. A. et al. A comparison of aspiration cytology and core needle biopsy according to tumor size of suspicious breast lesions. Diagn Cytopathol, v. 36, p. 26-31, 2008.

2. DeSantis, C. et al. Breast cancer statistics, 2011. CA Cancer J Clin, v. 61, p. 409-18, 2011.

3. GALHARDO, C. A.V. et al. Concordância entre core biopsy e exame anatomopatológico da peça cirúrgica em pacientes com câncer de mama. J Bras Patol Med Lab, v. 48, p. 59-65, 2012.

4. Linebarger, J. H. et al. Core needle biopsy rate for new cancer diagnosis in an interdisciplinary breast center: evaluation of quality of care 2007-2008. Ann Surg, v. 255, p. 38-43, 2012.

5. INCA. Tipos de câncer. Disponível em: <http://www2.inca.gov.br/wps/wcm/connect/tiposdecancer/site/home/mama/ cancer_mama++>. Acesso em: 14 fev. 2012. 